\title{
Effect of some Disturbance Factors on Falling Point Distribution of Unguided Rocket
}

\author{
H. Ban Le ${ }^{1}$ and P. Konečný 2* $^{*}$ \\ ${ }^{1}$ Le Quy Don Technical University, Hanoi, Vietnam \\ ${ }^{2}$ University of Defence, Brno, Czech Republic
}

\begin{abstract}
The manuscript was received on 5 October 2020 and was accepted after revision for publication as research paper on 16 May 2021.
\end{abstract}

\begin{abstract}
:
The paper investigates the individual and combined effects of disturbance factors including thrust misalignment, the offset of the centre of gravity and misalignment of the principal axes of inertia on falling point distribution of a type of unguided rocket (finstabilized rockets with single-stage solid-propellant rocket engines). The mathematical model used in the paper is developed from an available rocket motion model and solved for a representative rocket which is BM-21 rocket. The obtained results show dependencies of falling point deviation on disturbance parameters. These dependencies agree with the standard data given in the firing table; in addition, they are fuller and more insightful than the previous research.
\end{abstract}

\section{Keywords:}

CG offset, principal axes of inertia, thrust misalignment, unguided rocket

\section{Introduction}

Due to manufacturing and assembly errors, it is difficult for rockets to avoid thrust misalignment and mass distribution asymmetry. Thrust misalignment is understood as an angular deflection of the thrust vector towards the rocket's geometric symmetry axis. Mass distribution asymmetry is characterized by the offset of the centre of gravity (CG offset) and the principal axes of inertia (PAIs misalignment). The CG offset occurs when the rocket's CG is not on the rocket's geometric symmetry axis and there is a certain distance from its design position; PAIs are often designed to coincide with the rocket's geometric symmetry axis. PAIs misalignment occurs due to the uneven distribution of rocket mass along the length of a rocket.

\footnotetext{
* Corresponding author: Department of Weapons and Ammunition, University of Defence in Brno, Kounicova 156/65, CZ-662 10 Brno, Czech Republic. Phone: +420 9734420 37, E-mail: pavel.konecny@unob.cz. ORCID 0000-0003-0703-2315.
} 
These misalignments cause the rocket's falling points deviation from the standard falling point, so they affect the firing accuracy. The determination of the falling point distribution according to the disturbance parameters is important in giving technical requirements for the manufacturing process, ensuring that these misalignments are within allowable limits.

The effect of thrust misalignment and CG offset on rocket motion in the air is investigated in [1]. However, [1] mainly focuses on the mathematical model building and solving the model only in a narrow range of disturbance parameters. Therefore, the obtained results about the effect are incomplete and meagre.

In this paper, the mathematical model in [1] is developed to supplement the effect of PAIs misalignment on the rocket motion. In addition, the new model is solved more fully when investigating the entire variable range of the disturbance parameters. The combined effect of each pair of two or all three disturbance factors on the falling point distribution was done. This investigation is done for each firing angle and for the variable firing angle in the range from $5^{\circ}$ to $50^{\circ}$. The investigation results are significant in predicting the firing accuracy, so that the right rockets can be selected for the firing process.

\section{Mathematical Model of Unguided Rocket Motion in the Air Taking into Account Thrust Misalignment and Mass Distribution Asymmetry}

\subsection{Coordinate Systems}

For investigating the rocket motion in the air, some commonly used coordinate systems are as follows [2]:

- Normal earth coordinate system, $O_{\mathrm{g}} X_{\mathrm{g}} Y_{\mathrm{g}} Z_{\mathrm{g}}$

It is a fixed coordinate system on the ground with its origin $O_{\mathrm{g}}$ coinciding with the rocket's centre of gravity (rocket's CG) at the moment the rocket leave the launcher; the axis $O_{\mathrm{g}} X_{\mathrm{g}}$ is the intersection of the firing plane (the vertical plane containing the rocket axis when it starts to launch) with the horizontal plane across the origin. It is positive in the firing direction; the axis $O_{\mathrm{g}} Z_{\mathrm{g}}$ is perpendicular to $O_{\mathrm{g}} X_{\mathrm{g}}$ and downward; the axis $O_{\mathrm{g}} Y_{\mathrm{g}}$ is determined according to right rotation rule.

- Normal earth coordinate system attached to rocket, $O x_{\mathrm{g}} y_{\mathrm{g}} z_{\mathrm{g}}$

It has the origin in the rocket's $\mathrm{CG}, O$; its axes are always parallel with the axes of the normal earth coordinate system. $\left(O x_{\mathrm{g}}\left\|O_{\mathrm{g}} X_{\mathrm{g}} ; O y_{\mathrm{g}}\right\| O_{\mathrm{g}} Y_{\mathrm{g}} ; O z_{\mathrm{g}} \| O_{\mathrm{g}} Z_{\mathrm{g}}\right)$.

- Aerodynamic coordinate system, $O X_{\mathrm{a}} Y_{\mathrm{a}} Z_{\mathrm{a}}$

It is a coordinate system attached to the velocity vector of the rocket's CG; $O X_{\mathrm{a}}$ coincides with $v ; O Z_{\mathrm{a}}$ is perpendicular to $O X_{\mathrm{a}}$ in the plane of symmetry of the rocket and downward; $O Y_{\mathrm{a}}$ is perpendicular to the plane $O X_{\mathrm{a}} Z_{\mathrm{a}}$ in a right rotation rule. The position angles between the aerodynamic coordinate system and the normal earth coordinates system are: $\chi_{\mathrm{a}}-$ the aerodynamic azimuth, $\gamma_{\mathrm{a}}-$ the aerodynamic pitch and $\mu_{\mathrm{a}}-$ the aerodynamic roll angle.

- Body coordinate system, $O X Y Z$

It determines the position of the rocket axes. Its origin coincides with the rocket's $\mathrm{CG} ; O X$ is parallel to the rocket's geometric symmetry axis. In case the CG coincides with its design position on the geometric symmetry axis, then $O X$ coincides with the rocket's geometric symmetry axis; $O Z$ is perpendicular to $O X$ in the plane of symmetry of the rocket and downward; $O Y$ is determined according to right rotation rule. 
The rocket's spin motion is determined by the spin angle $v$ around the axis $O X$. The position angles between the body coordinate system and the aerodynamic coordinate system: $\alpha$ - the angle of attack (AOA) and $\beta$ - the sideslip angle (SSA).

Conventional unguided rockets are usually in axial symmetry, aerodynamic expressions almost do not change at spin angles of the rockets. Therefore, positions of the rockets in space can be determined through the coordinate systems above with the aerodynamic roll angle and roll angle are equal to zero. So, the axes $O Z_{\mathrm{a}}$ and $O Z_{\mathrm{a}}$ are in the vertical plane.

\subsection{Assumptions}

In the mathematical model, some assumptions are used as follows:

- in the moving process from launch, the rocket has only disturbances - thrust misalignment and mass distribution asymmetry,

- the rocket' CG deviates from its design position in the radial direction,

- when the rocket engine works, the rocket's centre of gravity moves in the parallel direction to the rocket axis $O X$,

- rocket engine operates stably, and the thrust does not change its direction during operation,

- the effects of wind, initial disturbance, Earth curvature, Magnus force and Coriolis force are ignored.

\subsection{Misalignment of Principal Axes of Inertia}

The rotational ability of a rocket is often characterized by the rocket's moments of inertia (rocket's MIs) with respect to its geometric axes that can be called rocket's GMIs. During rockets' production, mass distribution asymmetry causes deviations of the PAIs from their design positions - the geometric axes. This leads to rockets' GMIs being different from design values which are principal moments of inertia (PMIs).

Fig. 1 describes PAIs misalignment in a plane containing the geometric symmetry axis of a rocket, where $X_{\mathrm{I}} ; Y_{\mathrm{I}}$ denotes PAIs rotated from the geometric axes $O X$; $O Y_{\mathrm{s}}$ by a misalignment angle $\varepsilon_{\mathrm{I}}$. PMIs and GMIs of the rocket are $I_{\mathrm{p}} ; I_{\mathrm{e}}$ and $I_{x}, I_{y \mathrm{~s}}$, respectively.

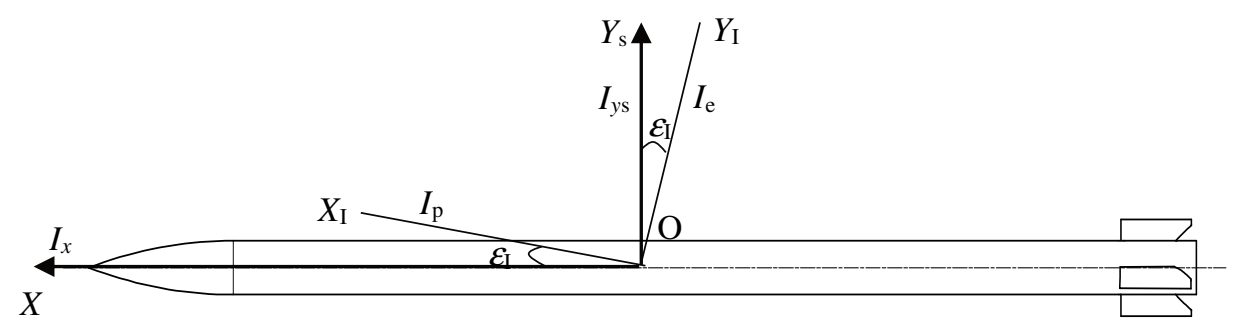

Fig. 1 PAIs misalignment in a symmetry plane of a rocket

The ratios $I_{x} / I_{\mathrm{p}}$ and $I_{y \mathrm{~s}} / I_{\mathrm{e}}$ of actual rockets are considered approximately equal to respective ratios of a uniform, congruent rocket model which can be made by mechanical design software such as Solidworks.

The misalignment angle $\varepsilon_{\mathrm{I}}$ is limited enough so that its effect on falling point deviations is clear and its impact on other disturbances can be evaluated. Through a few preliminary calculations, the chosen value of $\varepsilon_{\mathrm{I}}$ is: $\varepsilon_{\mathrm{I}} \leq 8^{\circ}$. With such PAIs misalign- 
ment angles, results of calculating the ratios $k_{x}=I_{x} / I_{\mathrm{p}}$ and $k_{y}=I_{y \mathrm{~s}} / I_{\mathrm{e}}$ are shown in Fig. 2.

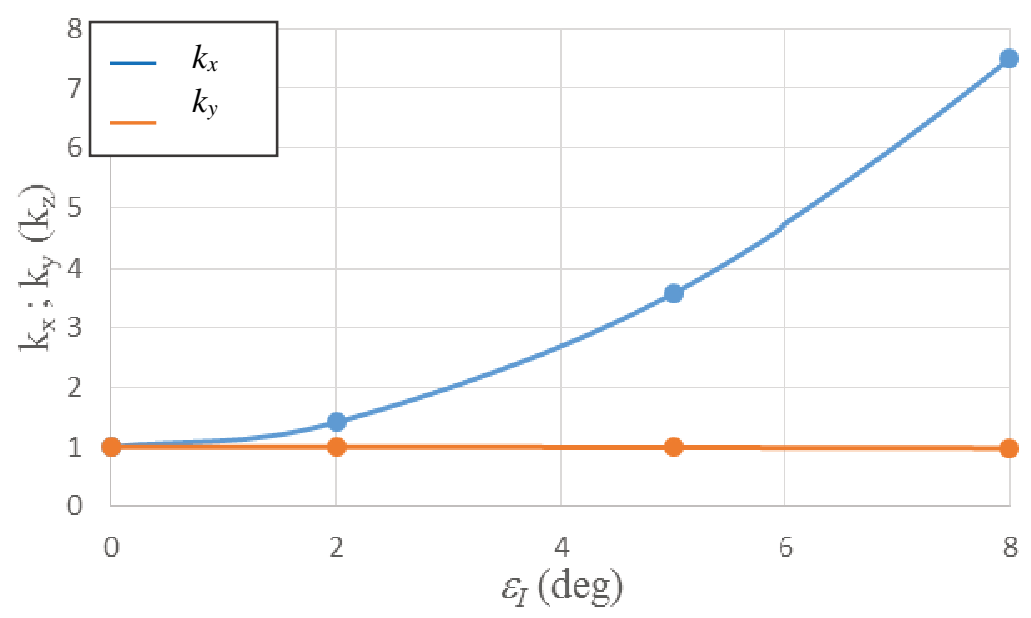

Fig. 2 Dependences of ratios $k_{x}$ and $k_{y}$ (or $k_{z}$ ) on PAIs misalignment angle

If only considering the PAIs misalignment in the plane $X O Y_{\mathrm{s}}$, rocket's MIs with respect to the axis $Z_{\mathrm{s}}$ which is perpendicular to the plane $X O Y_{\mathrm{s}}$ do not change. With such a calculation of the ratio of MI, then $k_{z}=1$. The roles of $Y_{\mathrm{s}}$ and $Z_{\mathrm{s}}$ are the same, so if only considering the PAIs misalignment in the plane $X O Z_{\mathrm{s}}$, then the ratios $k_{y}$ and $k_{z}$ above will swap values.

The PAIs misalignment in the plane $X O Y_{\mathrm{s}}$ above is shown in the body coordinate system as in Fig. 3, where $O X_{\mathrm{I}} Y_{\mathrm{I}} Z_{\mathrm{I}}$ is the PAIs system of the rocket.

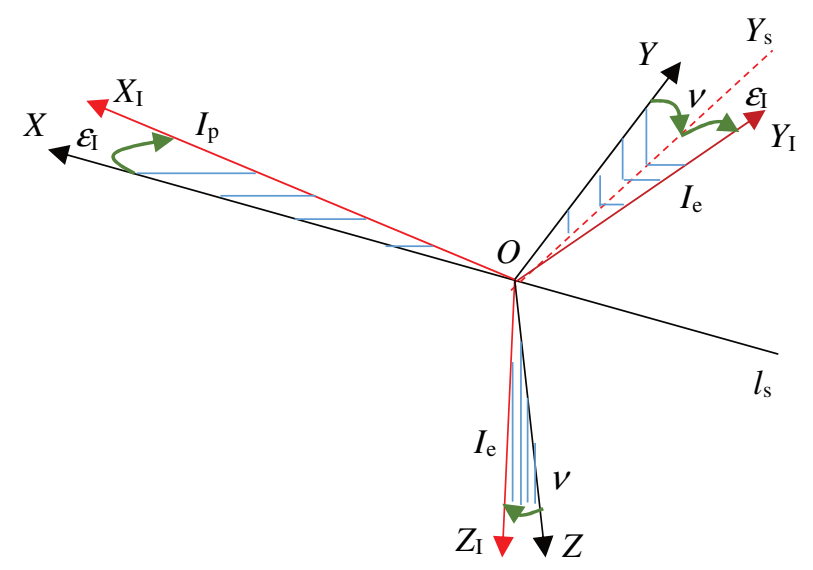

Fig. 3 A position of PAIs in the body coordinate system

According to Fig. 3, with constant values of $I_{\mathrm{e}} ; I_{\mathrm{p}}$ and $\varepsilon_{\mathrm{I}}$, the spin motion of the rocket leads to the simultaneous change of the rocket's MIs with respect to the coordinate axes $O Y$ and $O Z$. This change affects the rotational ability of the rocket in pitching and yawing oscillations, so affecting the range and lateral deviation, respectively. In the rocket's stable motion mode with the angles, the mutual effects between 
the pitching and yawing oscillation are small [2]. So, when only considering PAIs misalignment, the change of the rocket's MI with respect to the axis $O Y$ can be considered as the main cause of range deviation and the change of the rocket's MI with respect to the axis $O Z$ as the main cause of lateral deviation.

The rocket's MIs with respect to the axes $O Y$ and $O Z$ are approximately equal to the rocket's MIs with respect to the axis $O Y_{\mathrm{s}}$. So, changes of the rocket's MIs with respect to the axes $O Y$ and $O Z$ can be simulated as in the cases of PAIs misalignment occurred in the planes $O X Y$ and $O X Z$, respectively. From that, the effects of the MI changes on the deviations are determined.

Investigating all cases of inertia tensor is very complicated. The article only investigates PAIs misalignment (diagonal element of inertia tensor) for representing the change of the rocket's MI with respect to the axes $O Z$ (vertical), $O Y$ (horizontal) axes that affects the range and direction deviation greater than the other planes.

When the rocket has no CG offset, its PMIs at the initial time are $I_{\mathrm{p} 0}^{\prime}$ and $I_{\mathrm{e} 0}^{\prime}$. When considering CG offset, suppose that CG offset does not make changing PAIs misalignment angles, then at the initial time, PMIs are:

$$
\begin{aligned}
& I_{\mathrm{p} 0}=I_{\mathrm{p} 0}^{\prime}-m_{0} \cdot e_{\mathrm{m}}^{2} \\
& I_{\mathrm{e} 0}=I_{\mathrm{e} 0}^{\prime}
\end{aligned}
$$

where $m_{0}-$ the rocket's initial mass.

The rocket's MIs with respect to the axes $O X ; O Y ; O Z$ are denoted as $I_{x 0} ; I_{y 0} ; I_{z 0}$, respectively. For PAIs misalignment in two planes $O X Y$ and $O X Z$, these MIs at the initial time are determined as follows:

PAIs misalignment in the plane $O X Y$ :

$$
\begin{aligned}
& I_{x 0}=k_{x} I_{\mathrm{p} 0} \\
& I_{y 0}=k_{y} I_{\mathrm{e} 0} \\
& I_{z 0}=I_{\mathrm{e} 0}
\end{aligned}
$$

PAIs misalignment in the plane $O X Z$ :

$$
\begin{aligned}
I_{x 0} & =k_{x} I_{\mathrm{p} 0} \\
I_{y 0} & =I_{\mathrm{e} 0} \\
I_{z 0} & =k_{z} I_{\mathrm{e} 0}
\end{aligned}
$$

where $k_{z}=k_{y}$.

\subsection{Mathematical Model}

According to [1], characteristic parameters of CG offset and thrust misalignment in the body coordinate system are shown in Fig. 4.

In Fig. 4: $l_{\mathrm{s}}$ - the rocket's geometric symmetry axis; $O^{\prime}-$ the design position of CG; $O_{\mathrm{T}}-$ the distributed centre of motor nozzles' critical sections; $\delta_{\mathrm{T}}-$ the distance between $O^{\prime}$ and $O_{\mathrm{T}} ; \lambda_{\mathrm{T}} ; \varepsilon_{\mathrm{T}}-$ the thrust misalignment angle and direction angle of thrust misalignment; $e_{\mathrm{m}} ; \varepsilon_{\mathrm{m}}-$ the CG offset and direction angle of CG offset; $D_{\mathrm{T}}-$ the intersection between thrust and the rocket's cross-section at the CG. At the initial time, the position of CG is $O_{\mathrm{D} 0} \equiv \mathrm{O}$, the intersection is $D_{\mathrm{T} 0}$. These points characterize the disturbance factors at the initial time and they are called feature points.

When adding PAIs misalignment, the mathematical model in [1] is developed into a new model, which is the mathematical model of rocket motion taking into account 
thrust misalignment, CG offset, and PAIs misalignment. From [1], Eqs (2) and (3), the mathematical model is rewritten as follows:

$$
\begin{aligned}
\dot{V}= & \frac{1}{m}\left\{T \cos \lambda_{\mathrm{T}} \cos \alpha \cdot \cos \beta+T \sin \lambda_{\mathrm{T}}\left[\cos \left(\varepsilon_{\mathrm{T}}+v\right) \sin \alpha+\right.\right. \\
& \left.\left.+\sin \left(\varepsilon_{T}+v\right) \cos \alpha \cdot \sin \beta\right]-D-m g \sin \gamma_{a}\right\} \\
\dot{\gamma}_{a}= & \frac{1}{m v}\left\{T \cos \lambda_{\mathrm{T}} \sin \alpha \cdot \cos \beta-T \sin \lambda_{\mathrm{T}}\left[\cos \left(\varepsilon_{\mathrm{T}}+v\right) \cos \alpha-\right.\right. \\
& \left.\left.-\sin \left(\varepsilon_{\mathrm{T}}+v\right) \sin \alpha \cdot \sin \beta\right]+L-m g \cos \gamma_{\mathrm{a}}\right\} \\
\dot{\chi}_{a}= & \frac{1}{m v \cos \gamma_{\mathrm{a}}}\left[T \cos \lambda_{\mathrm{T}} \cdot \sin \beta-T \sin \lambda_{\mathrm{T}} \cdot \sin \left(\varepsilon_{\mathrm{T}}+v\right) \cos \beta+Y_{\mathrm{L}}\right] \\
\dot{\alpha}= & \frac{1}{\cos \beta}\left[\omega_{y}-\dot{\gamma}_{\mathrm{a}}\left(\cos \beta \cdot \cos \chi_{\mathrm{a}}-\cos \alpha \cdot \sin \beta \cdot \sin \chi_{\mathrm{a}}\right)-\dot{\chi}_{\mathrm{a}} \sin \alpha \cdot \sin \beta\right] \\
\dot{\beta}= & \omega_{z}-\dot{\gamma}_{\mathrm{a}} \sin \alpha \cdot \sin \chi_{\mathrm{a}}-\dot{\chi}_{\mathrm{a}} \cos \alpha \\
\dot{v}= & \omega_{x}-\dot{\alpha} \sin \beta-\dot{\gamma}_{\mathrm{a}}\left(\cos \alpha \cdot \cos \beta \cdot \sin \chi_{\mathrm{a}}+\sin \beta \cdot \cos \chi_{\mathrm{a}}\right)+\dot{\chi}_{\mathrm{a}} \sin \alpha \cdot \cos \beta \\
\dot{\omega}_{x}= & \frac{1}{I_{x}}\left[-T \sin \lambda_{\mathrm{T}} e_{\mathrm{m}} \sin \left(\varepsilon_{\mathrm{T}}+v\right) \cos \left(v+\varepsilon_{m}\right)+T \sin \lambda_{\mathrm{T}} e_{\mathrm{m}} \cos \left(\varepsilon_{\mathrm{T}}+v\right) \sin \left(v+\varepsilon_{\mathrm{m}}\right)+L_{\mathrm{R}}\right] \\
\dot{\omega}_{y}= & \frac{1}{I_{y}}\left[-T \cos \lambda_{\mathrm{T}} e_{\mathrm{m}} \cos \left(v+\varepsilon_{\mathrm{m}}\right)+T \sin \lambda_{\mathrm{T}} \cos \left(\varepsilon_{\mathrm{T}}+v\right) \delta_{\mathrm{T}}-\left(I_{x}-I_{z}\right) \omega_{x} \omega_{z}-I_{z} \omega_{z} \dot{v}+M\right] \\
\dot{\omega}_{z}= & \frac{1}{I_{z}}\left[-T \cos \lambda_{\mathrm{T}} e_{\mathrm{m}} \sin \left(v+\varepsilon_{\mathrm{m}}\right)+T \sin \lambda_{\mathrm{T}} \sin \left(\varepsilon_{\mathrm{T}}+v\right) \delta_{\mathrm{T}}-\left(I_{y}-I_{x}\right) \omega_{x} \omega_{y}+I_{y} \omega_{y} \dot{v}+N\right] \\
\dot{X}_{g}= & v \cos \gamma_{\mathrm{a}} \cdot \cos \chi_{\mathrm{a}} \\
\dot{Y}_{g}= & v \cos \gamma_{\mathrm{a}} \cdot \sin \chi_{\mathrm{a}} \\
\dot{Z}_{g}= & -v \sin \gamma_{\mathrm{a}} \\
I_{x}= & k_{x} I_{p 0} \\
I_{y}= & k_{y} I_{e 0} \\
I_{z}= & k_{z} I_{e 0}
\end{aligned}
$$

where $m$ - the rocket's instantaneous mass; $v$ - the rocket's velocity; $T$ - the thrust; $L$; $D ; Y_{\mathrm{L}} ; M ; L_{\mathrm{R}} ; N$ - the aerodynamic forces and moments; $I_{x} ; I_{y} ; I_{z}$ - the rocket's MI with respect to axes $O X, O Y$ and $O Z$, respectively; $\omega_{x} ; \omega_{y} ; \omega_{z}-$ the rocket's angular velocity with respect to axes $O X, O Y$ and $O Z$, respectively; $k_{x} ; k_{y} ; k_{z}-$ the coefficients dependent on PAIs misalignment angle; $k_{y}=1$ when PAIs misalignment in the plane $O X Z ; k_{z}=1$ when PAIs misalignment in the plane $O X Y$;

For solving the mathematical model, a used approximation method is the fourthorder Runge-Kutta. For ensuring the accuracy of calculated results, the integral step is assigned decreasing values by dividing by 2 . This is done continuously until the range deviation corresponding to two consecutive division steps is less than 1/100000 of range.

\section{Effect of Disturbance Factors on Falling Point Distribution}

The mathematical model is solved for BM-21 rocket, which represents the type of unguided, fin-stabilized rockets with single-stage solid-propellant rocket engines. The obtained results can be applied to other rockets of this type. The ballistic parameters and aerodynamic coefficients are taken from $[1,3]$. The results of solving the mathe- 
matical model show the dependence of the falling point deviation on the disturbance factors which are presented in the following sections.

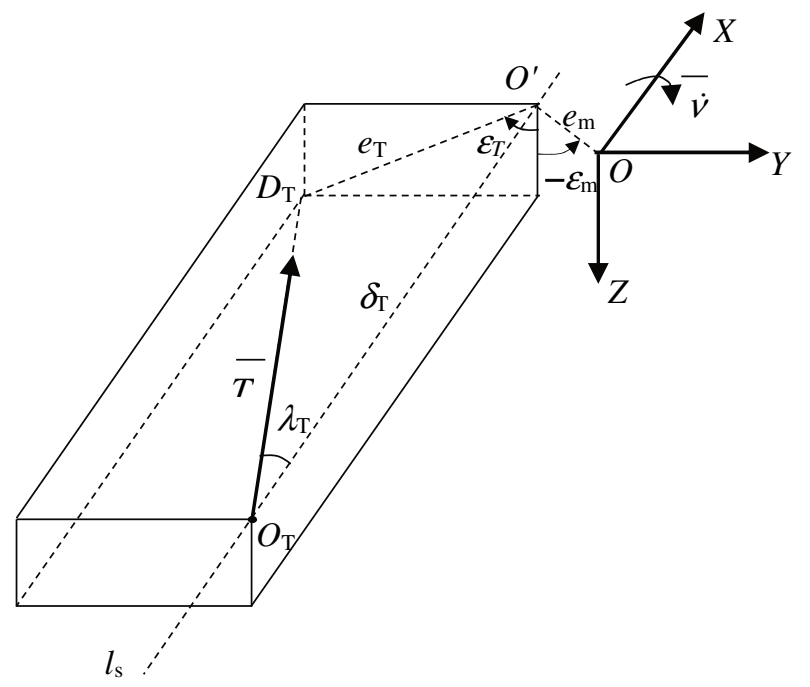

Fig. 4 A position of PAIs in the body coordinate system

\subsection{Effect of Thrust Misalignment}

According to [1], for each firing angle, the falling point deviation depends linearly on thrust misalignment angle at the initial time $\lambda_{\mathrm{T} 0}$ and like sinusoidal on the direction angle of thrust misalignment at the initial time $\varepsilon_{\mathrm{T} 0}$; deviation graphs get extremes at values of $\varepsilon_{\mathrm{T} 0}$ that are approximately equal to $0^{\circ} ; 90^{\circ} ; 180^{\circ}$ and $270^{\circ}$. These results show that for each angle $\lambda_{\mathrm{T} 0}$ and angles $\varepsilon_{\mathrm{T} 0}=0^{\circ} ; 90^{\circ} ; 180^{\circ}$ or $270^{\circ}$, the corresponding falling point deviations are most likely to exceed the allowable limit. So, it is possible to determine the limit value of $\lambda_{\mathrm{T} 0}$ through determining its limit corresponding to angle $\varepsilon_{\mathrm{T} 0}=0^{\circ} ; 90^{\circ} ; 180^{\circ}$ and $270^{\circ}$.

With any theoretical value of thrust misalignment angle at the initial time, such as $\lambda_{\mathrm{T} 0}=0.001 \mathrm{rad}$, its effects on the range and lateral deviation for each firing angle are presented in [1]. For variable firing angle, the effects on deviations corresponding to the direction angles: $\varepsilon_{\mathrm{T} 0}=0^{\circ} ; 90^{\circ} ; 180^{\circ}$ and $270^{\circ}$ are shown in Figs 5 and 6 .

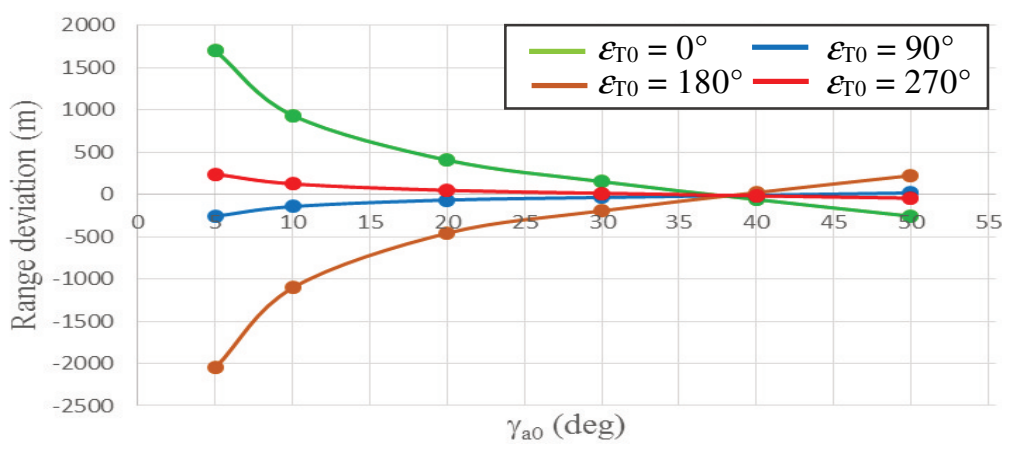

Fig. 5 Range deviation according to firing angles when $\lambda_{\mathrm{T} 0}=0.001 \mathrm{rad}$ 


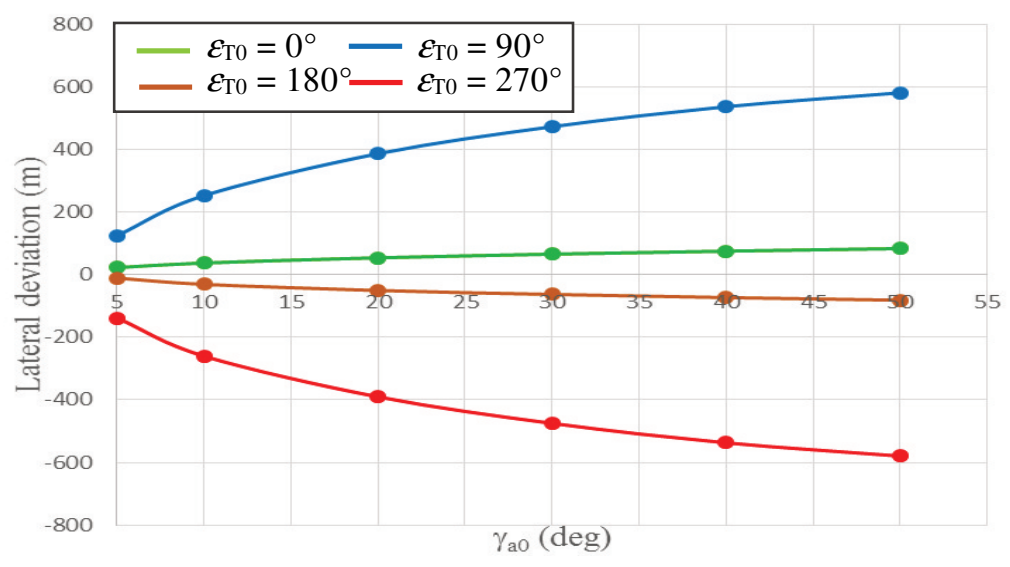

Fig. 6 Lateral deviation according to firing angles when $\lambda_{\mathrm{T} 0}=0.001 \mathrm{rad}$

At the direction angles $\varepsilon_{\mathrm{T} 0}=0^{\circ} ; 90^{\circ} ; 180^{\circ}$ and $270^{\circ}$, by changing the values of $\lambda_{\mathrm{T} 0}$, the corresponding falling point deviations are compared with the allowable limits in the firing table. Then it is possible to determine the limits of $\lambda_{\mathrm{T} 0}$ for variable firing angle, named $\lambda_{\text {Tmax }}$ and shown in Fig. 7.

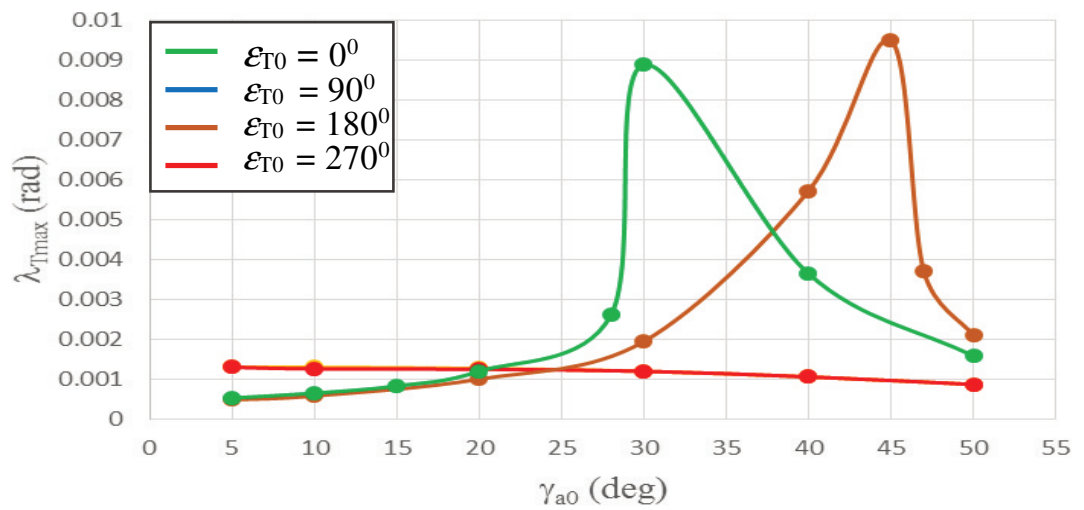

Fig. 7 Dependence of maximum thrust misalignment angle on firing angle

\section{Discussion:}

- from Figs 5 and 6, it can be found that: when the firing angle increases, the lateral deviations increase too. The graphs of the range deviation almost intersect at firing angle $\gamma_{\mathrm{a} 0}=38^{\circ}$; in interval $\gamma_{\mathrm{a} 0}$ from $5^{\circ}$ to $38^{\circ}$, the range deviations decrease; when $\gamma_{\mathrm{a} 0} \geq 38^{\circ}$ the range deviations gradually increase and change the sign. The sign change in range deviations due to firing ranges reach the maximum values, then reduce when the firing angle increases. If drag is not taken into account, the firing angle corresponding to the maximum range is $45^{\circ}$, but in fact, drag reduces this angle. When the parameter $\lambda_{\mathrm{T} 0}$ changes, the intersection point moves insignificantly,

- from Fig. 7, the limits of $\lambda_{\mathrm{T} 0}$ can be used in production as the tolerances of actual deviations. That is, the actual thrust misalignment angle at the initial time 
must be within is in these limits plus a constant which depends on technological capabilities. The limits of $\lambda_{\text {T0 }}$ are also applied in the selection of solutions for the technology, for example for firing angles from 30 to 35 , it is recommended to make the direction angle $\varepsilon_{\mathrm{T} 0}$ approximate to 0 to get a large production tolerance domain, ensuring the firing accuracy.

- the laws of range deviation and lateral deviation with firing angle changes within $5^{\circ} \div 38^{\circ}$ are consistent law of falling point dispersion $[4,5]$, as Fig. 8:

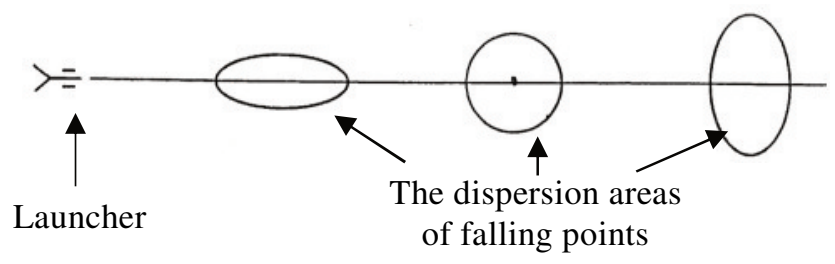

Fig. 8 Dispersion law of falling points [4]

Fig. 8 shows: at small firing angles, the range deviation is greater than the direction deviation; at great firing angles, the range deviation is smaller than the direction deviation; when the firing angle increases, the range deviation decreases, but direction deviation increases gradually.

The range deviations with respect to angles $\mathcal{E}_{\mathrm{T} 0}=0^{\circ} ; 180^{\circ}$ are greater than angles $\varepsilon_{\mathrm{T} 0}=90^{\circ} ; 270^{\circ}$ but the lateral deviations are smaller. This is because, at the initial time, the thrust moments in the plane $O X Z$ corresponding to angles $\varepsilon_{\mathrm{T} 0}=0^{\circ} ; 180^{\circ}$ are greater but in the plane $O X Y$, they are smaller.

\subsection{Effect of CG Offset}

For each firing angle, the dependence of the falling point deviation on parameters $e_{m} 0$ and $\varepsilon_{\mathrm{m} 0}$ is as the parameters $\lambda_{\mathrm{T} 0}$ and $\varepsilon_{\mathrm{T} 0}$, respectively.

When firing angle changes, the effect of CG offset on falling point deviation corresponding to the disturbance parameters: $e_{\mathrm{m} 0}=0.001 \mathrm{~m} ; \varepsilon_{\mathrm{m} 0}=0^{\circ} ; 90^{\circ} ; 180^{\circ}$ and $270^{\circ}$ are shown in Figs 9 and 10.

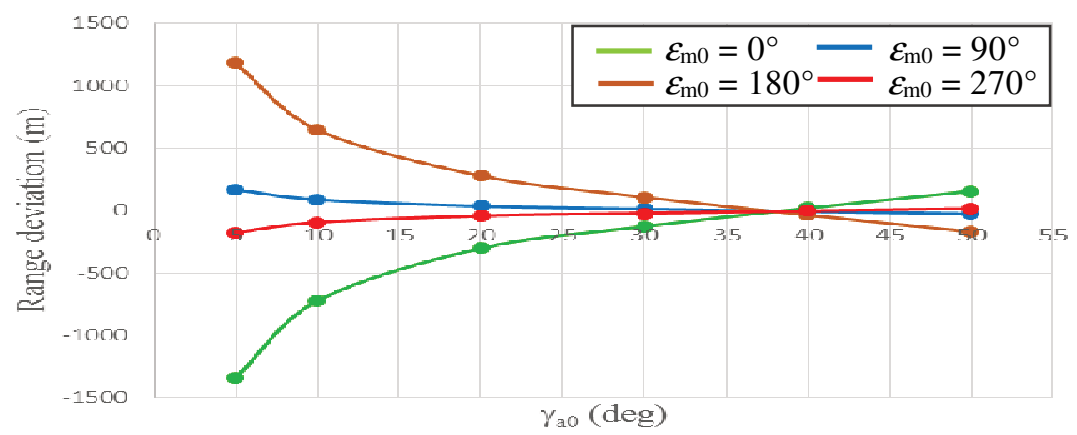

Fig. 9 Range deviation according to firing angles when $e_{\mathrm{m} 0}=0.001 \mathrm{~m}$ 


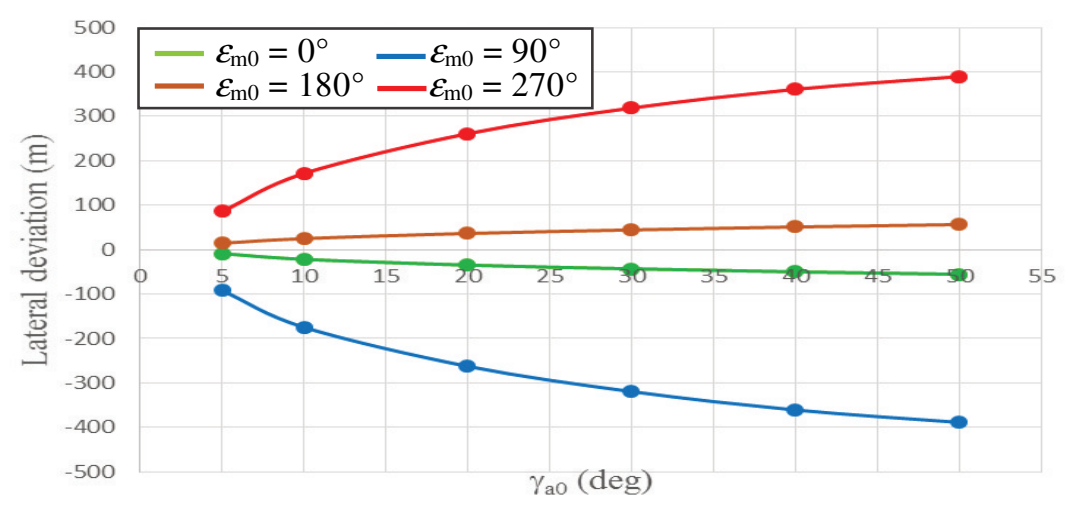

Fig. 10 Lateral deviation according to firing angles when $e_{\mathrm{m} 0}=0.001 \mathrm{~m}$

\section{Discussion}

The comparison of Figs 5 and 9; Figs 6 and 10, shows that with the same relative distance between the two points $D_{\mathrm{T} 0}$ and $O_{\mathrm{D} 0}$ (Fig 4), the effect characteristics of CG offset are almost similar to the effect characteristics of thrust misalignment, as:

- if disturbance angle $\left(\varepsilon_{\mathrm{m} 0}\right.$ or $\left.\varepsilon_{\mathrm{T} 0}\right)$ has positions which are symmetrical through the centre of geometric symmetry $O^{\prime}$, then the corresponding deviation graphs are also almost symmetric through the 0 -deviation axis. That is because the magnitudes of forces and moments corresponding to the symmetrical positions are the same, but of opposite signs. The slight difference is caused by the gravity at the initial time,

- when the firing angle increases, the lateral deviations increase; the range deviations decrease with $\gamma_{\mathrm{a} 0}=5^{\circ} \div 38^{\circ}$, then they gradually increase and change sign,

- the deviation graphs corresponding to the angles $\varepsilon_{\mathrm{T} 0}$ and $\mathcal{E}_{\mathrm{m} 0}=\varepsilon_{\mathrm{T} 0}+180^{\circ}$ are nearly the same.

\subsection{Effect of Misalignment of Principal Axes of Inertia}

When investigating the effect of PAIs misalignment, thrust misalignment and CG offset are ignored.

\section{a) For each firing angle}

The characteristic of the effect can be shown by investigating the motions as below, with a specific firing angle of $30^{\circ}$.

The investigated motions have the following firing conditions:

- $v_{0}=40 \mathrm{~m} / \mathrm{s} ; \gamma_{\mathrm{a} 0}=30^{\circ} ; \dot{v}_{0}=0.02 \mathrm{rad} / \mathrm{s} ; \alpha_{0}=\beta_{0}=0 ; \dot{\chi}_{\mathrm{a} 0}=\dot{\gamma}_{\mathrm{a} 0}=0 ; \varepsilon_{\mathrm{I} 0} \leq 8$.

The calculation results show the dependence of the range deviation and the lateral deviation on the PAIs misalignment angle in two planes $O X Y$ and $O X Z$, as shown in Fig. 11.

\section{Discussion}

In Fig. 11, the range deviation is almost constant; lateral deviation increases in the negative direction.

In two cases of PAIs misalignment, MIs relative to longitudinal axes (longitudinal-MI) are the same, but MIs relative to horizontal axes (horizontal-MI) are different. According to Fig. 11, corresponding deviations in the two cases are the same, and the range deviation is very small compared to the lateral deviation. This shows that hori- 
zontal-MI has little effect on deviations; longitudinal-MI has a great effect on lateral deviation and little effect on range deviation at this firing angle.

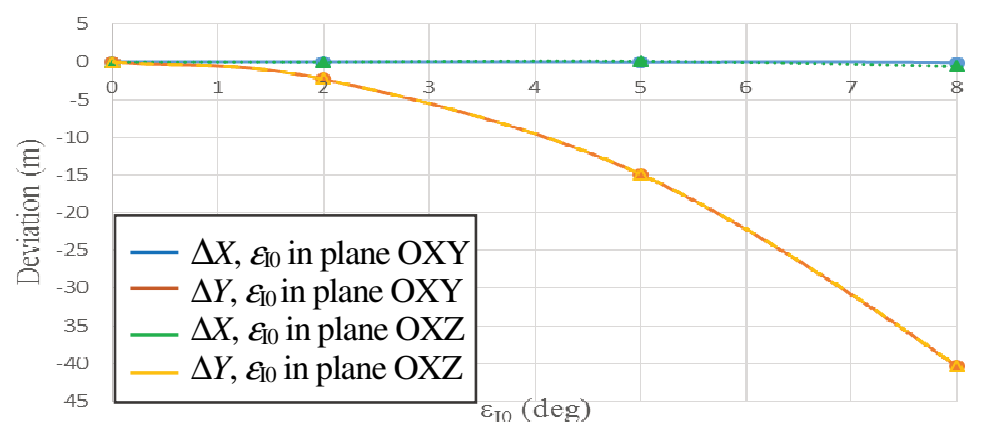

Fig. 11 Dependence of falling point deviation on the PAIs misalignment angle $\varepsilon_{10}$

\section{b) For variable firing angle}

The results in Fig. 11 show that the effects of PAIs misalignment in the two planes $O X Y$ and $O X Z$ are almost the same. Investigated results of the falling point deviation at the firing angles when PAIs misalignment angle $\varepsilon_{10}=5^{\circ}$ (in the plane $O X Y$ or $O X Z$ ) are shown in Fig. 12.

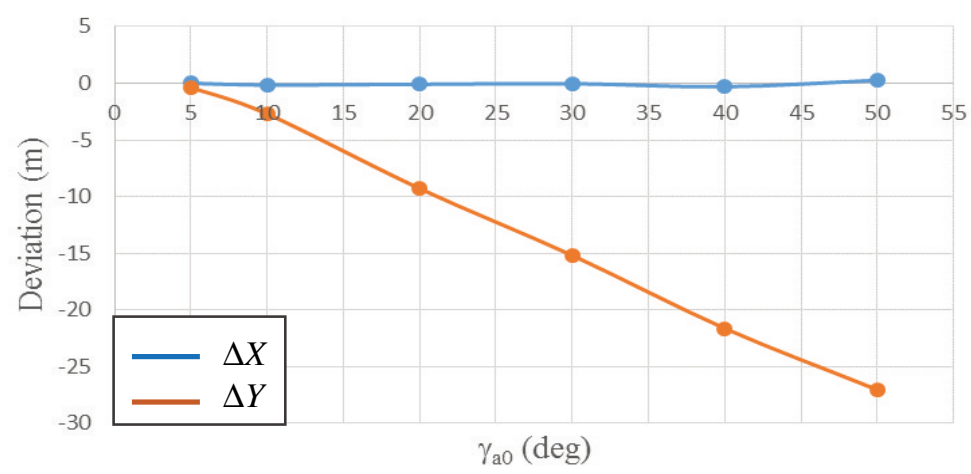

Fig. 12 Dependence of range deviation and lateral deviation on firing angle when the $P A I$ s misalignment angle $\varepsilon_{10}=5^{\circ}$ and in the plane $O X Y$

\subsection{Combined Effect of Thrust Misalignment and CG Offset}

When considering the combined effect of thrust misalignment and CG offset, PAIs misalignment is ignored.

As in Fig. 4, the two disturbances are likely to appear at every position within their limits, which leads to a multitude of relative positions between CG and thrust.

There are relative positions that ensure the stability of the rocket motion but there are also positions that make the rocket motion unstable. A more important problem is to determine the relative positions between the disturbances that cause the greatest deviations, called the dangerous points. From that, it is possible to determine the safest conditions for disturbance parameters to ensure firing accuracy at each firing angle. 
The conclusions in [1] show that, in the case of individual disturbances only, the most dangerous cases occur when disturbance angles $\varepsilon_{\mathrm{T} 0}\left(\right.$ or $\left.\varepsilon_{\mathrm{m} 0}\right)$ are $0^{\circ} ; 90^{\circ} ; 180^{\circ}$; $270^{\circ}$, meaning the relative positions of feature points of thrust and CG are in either horizontal or vertical direction. However, when there is a combination of both factors, that conclusion may not be right. Therefore, it is necessary to redefine the dangerous points.

Fig. 13 shows positions of two feature points of thrust and CG in the combination of these disturbances, where the relative position of point $D_{\mathrm{T} 0}$ with respect to point $O_{\mathrm{D} 0}$ is determined by the parameters $r$ and $\varepsilon_{\mathrm{r}}$.

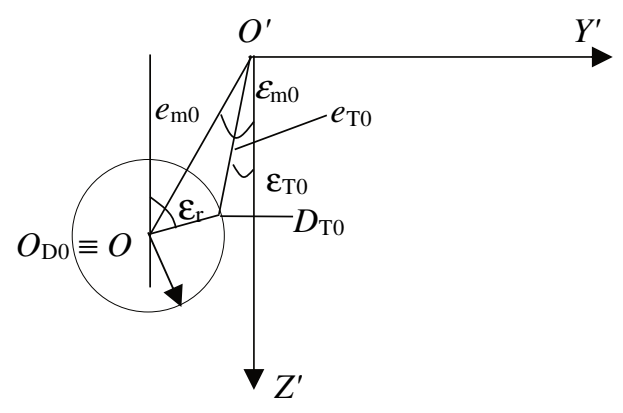

Fig. 13 Geometric relationship between two the feature points $D_{\mathrm{T} 0}$ and $O_{\mathrm{D} 0}(\equiv O)$

In Fig. 13, the CG, $O$ deviates from its ideal position, $O$ ', the coordinate system $O^{\prime} X^{\prime} Y^{\prime} Z^{\prime}$ is the ideal position of the coordinate system $O X Y Z$. Parameters of thrust misalignment are determined by the formula:

$$
\begin{aligned}
& e_{\mathrm{T} 0}=\sqrt{e_{\mathrm{m} 0}^{2}+r^{2}-2 e_{\mathrm{m} 0} r \cos \left(\varepsilon_{r}-\varepsilon_{\mathrm{m} 0}\right)} ; \lambda_{\mathrm{T} 0}=\arctan \frac{e_{\mathrm{T} 0}}{\delta_{0}} \\
& \varepsilon_{\mathrm{T} 0}=\varepsilon_{\mathrm{m} 0}-\arcsin \left[\frac{r}{e_{\mathrm{T} 0}} \sin \left(\varepsilon_{r}-\varepsilon_{\mathrm{m} 0}\right)\right]
\end{aligned}
$$

With initial firing conditions are: $v_{0}=40 \mathrm{~m} / \mathrm{s} ; \gamma_{\mathrm{a} 0}=30^{\circ} ; \dot{v}_{0}=0.02 \mathrm{rad} / \mathrm{s}$; $\alpha_{0}=\beta_{0}=0 ; \chi_{\mathrm{a} 0}=\gamma_{\mathrm{a} 0}=0$, disturbances are investigated corresponding to the three cases as following:

- $e_{\mathrm{m} 0}=0.0015 \mathrm{~m} ; \varepsilon_{\mathrm{m} 0}=0^{\circ} ; r=0.0005 \mathrm{~m} ; \varepsilon_{r}=0^{\circ} ; 30^{\circ} \ldots 300^{\circ} ; 330^{\circ}$,

- $e_{\mathrm{m} 0}=0.0015 \mathrm{~m} ; \varepsilon_{\mathrm{m} 0}=30^{\circ} ; r=0.0005 \mathrm{~m} ; \varepsilon_{r}=0^{0} ; 30^{\circ} \ldots 300^{\circ} ; 330^{\circ}$,

- $e_{\mathrm{m} 0}=0.0015 \mathrm{~m} ; \varepsilon_{\mathrm{m} 0}=90^{\circ} ; r=0.0005 \mathrm{~m} ; \varepsilon_{r}=0^{\circ} ; 30^{\circ} \ldots 300^{\circ} ; 330^{\circ}$.

Range deviations of three cases above are shown in Fig. 14; lateral deviations are shown in Fig. 15.

The results in Figs 13 and 14 show that the deviation graphs are almost the same. The two graphs at the boundary positions correspond to angles $\varepsilon_{\mathrm{m} 0}=0^{\circ}$ and $90^{\circ}$. Other calculations also indicate that the deviation graphs get boundary positions at the angles of $\varepsilon_{\mathrm{m} 0}=180^{\circ}$ and $270^{\circ}$.

\section{Discussion}

For a certain value of the angle $\mathcal{E}_{\mathrm{m} 0}$, the deviation graph gets extremes at angles $\mathcal{E}_{r}=0^{\circ} ; 90^{\circ} ; 180^{\circ}$ and $270^{\circ}$, corresponding to the segment $O D_{\mathrm{T} 0}$ in the vertical or horizontal plane. This result is consistent with the result of the relative positions of two points $O$ (at the initial time) and $D_{\mathrm{T} 0}$ as investigated in [1]. 
According to Figs 13 and 14, when $\varepsilon_{\mathrm{m} 0}$ changes, with the same distance $O D_{\mathrm{T} 0}$, the deviation graphs differ very little and reach the boundary positions at angles $\varepsilon_{\mathrm{m} 0}=0^{\circ}$; $90^{\circ}$. Other calculation results are similar for $\varepsilon_{\mathrm{m} 0}=180^{\circ} ; 270^{\circ}$. From that, the falling point deviations are maximum when the segment $O D_{\mathrm{T} 0}$ is on the plane $O^{\prime} X^{\prime} Y^{\prime}$ or $O^{\prime} X^{\prime} Z^{\prime}$.

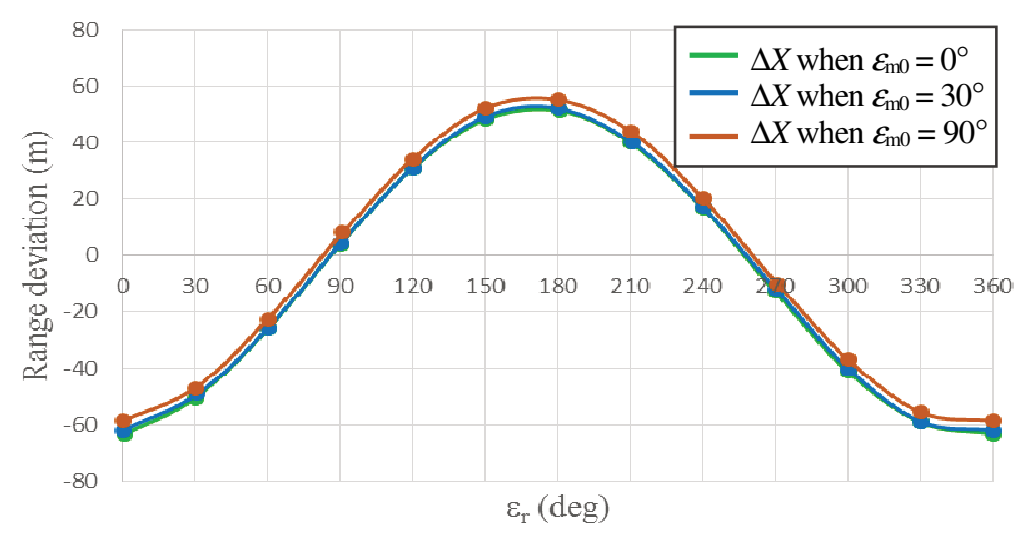

Fig. 14 Dependence of range deviation on the angle $\mathcal{E}_{r}$ when $\varepsilon_{\mathrm{m} 0}=0^{\circ} ; 30^{\circ}$ and $90^{\circ}$

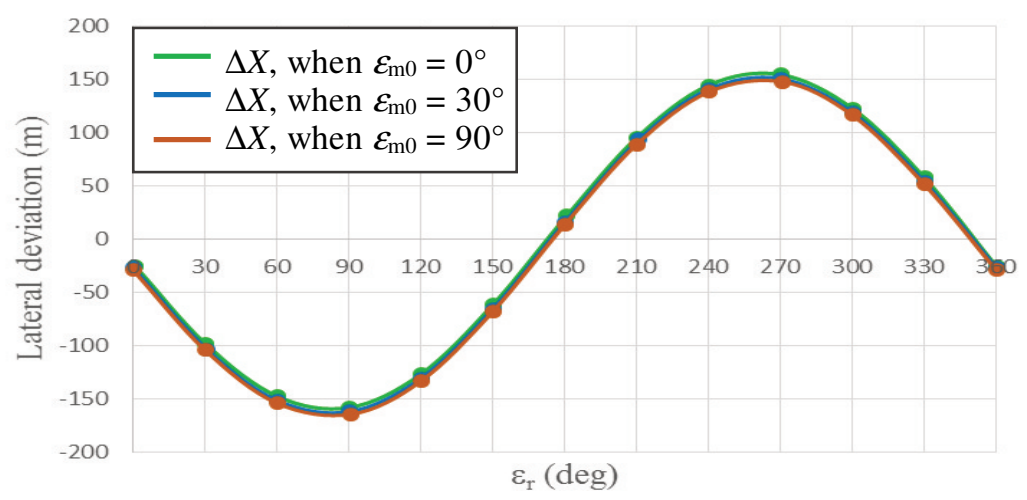

Fig. 15 Dependence of lateral deviation on the angle $\varepsilon_{r}$ when $\varepsilon_{\mathrm{m} 0}=0^{\circ} ; 30^{\circ}$ and $90^{\circ}$

\subsection{Combined Effect of Thrust Misalignment and Misalignment of Principal Axes of Inertia}

In the paper, the combination of PAIs misalignment with two remaining disturbances is studied to show the impact of PAIs misalignment on the effect of thrust misalignment or CG offset on falling point deviation. When investigating the combination of thrust misalignment and PAIs misalignment, CG offset is ignored.

a) For each firing angle

The characteristic of the effect can be shown by investigating the motions as below, with a specific firing angle of $30^{\circ}$.

Investigating rocket motions containing thrust misalignment and PAIs misalignment can be expressed as follows: 
- $v_{0}=40 \mathrm{~m} / \mathrm{s} ; \gamma_{\mathrm{a} 0}=30^{\circ} ; \dot{v}_{0}=0.02 \mathrm{rad} / \mathrm{s} ; \alpha_{0}=\beta_{0}=0 ; \dot{\chi}_{\mathrm{a} 0}=\dot{\gamma}_{\mathrm{a} 0}=0$;

- $\lambda_{\mathrm{T} 0}=0.001 \mathrm{rad}, \varepsilon_{\mathrm{T} 0}=0^{\circ}$; PAIs misalignment is in the planes $O X Y$ or $O X Z$ with a misalignment angle of $\varepsilon_{10} \leq 8^{\circ}$.

Dependence of the range deviation and the lateral deviation on the PAIs misalignment angle in two planes $O X Y$ and $O X Z$ are shown in Fig. 16.

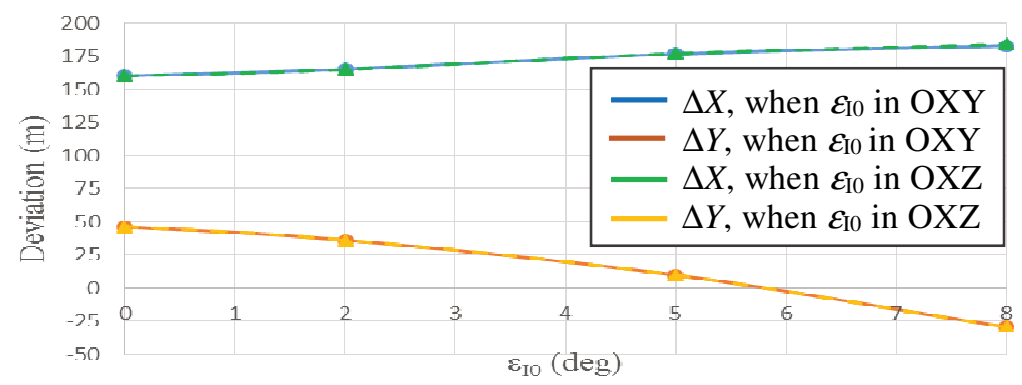

Fig. 16 Dependence of falling point deviation on the PAIs misalignment angle $\varepsilon_{0}$ when

$$
\lambda_{\mathrm{T} 0}=0.001 \mathrm{rad} ; \varepsilon_{\mathrm{T} 0}=0^{\circ}
$$

Fig. 16 shows that corresponding deviations in the two cases of PAIs misalignment are the same. Comparing with the results in Fig. 11, it is shown that the impacts of two cases of PAIs misalignment on the effect of thrust misalignment are the same.

b) For variable firing angle

Investigating the motions similar to those in section 3.1: $\gamma_{\mathrm{a} 0}$ changes; $\lambda_{\mathrm{T} 0}=0.001 \mathrm{rad}$; $\varepsilon_{\mathrm{T} 0}=0^{\circ} ; 90^{\circ} ; 180^{\circ}$ and $270^{\circ}$, but there is an additional disturbance factor as PAIs misalignment angle $\varepsilon_{10}=5^{\circ}$. The results of comparing falling point deviations between motions with and without PAIs misalignment are shown in Figs 17-20.

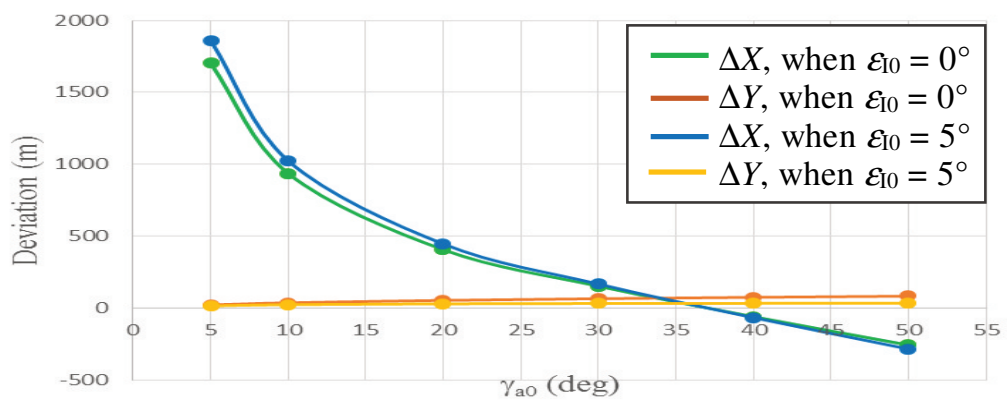

Fig. 17 Dependence of falling point deviation on firing angle when $\lambda_{\mathrm{T} 0}=0.001 \mathrm{rad}$;

$$
\varepsilon_{\mathrm{T} 0}=0^{\circ} ; \varepsilon_{\mathrm{I} 0}=0^{\circ} \text { and } 5^{\circ}
$$

\section{Discussion}

- The figures above show that PAIs misalignment has changed the effect of thrust misalignment on falling point deviation.

- Comparing pairs of Figs 16 and 18; Figs 17 and 19, they show that the impact of PAIs misalignment (when combined with thrust misalignment) on the effect of thrust misalignment about range deviation corresponding to symmetrical disturbance angles $\varepsilon_{\mathrm{T} 0}: 0^{\circ}$ and $180^{\circ} ; 90^{\circ}$ and $270^{\circ}$ are almost the same. With small firing angles $\left(\leq 25^{\circ}\right)$, when $\varepsilon_{\mathrm{T} 0}=0^{\circ}$ and $180^{\circ}$, the range deviation increases; 


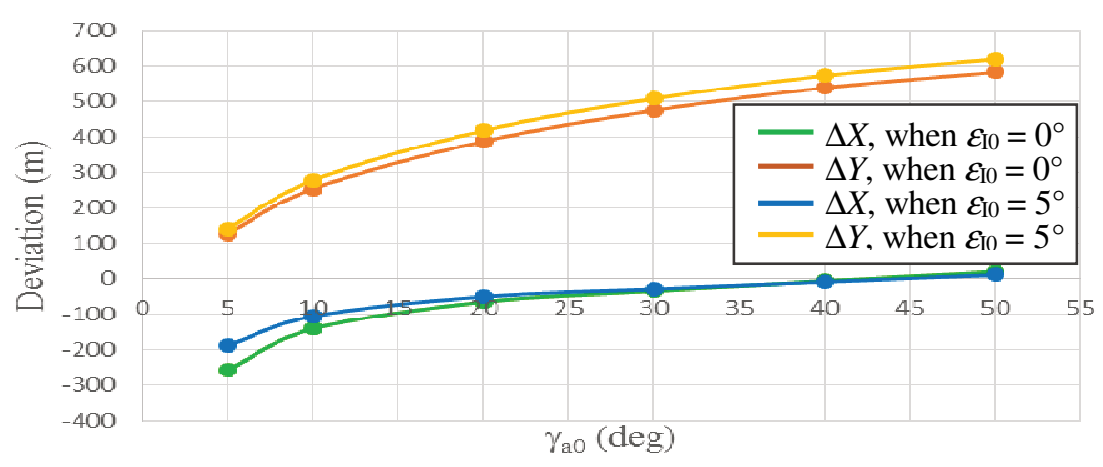

Fig. 18 Dependence of falling point deviation on firing angle when $\lambda_{\mathrm{T} 0}=0.001 \mathrm{rad} ; \varepsilon_{\mathrm{T} 0}=90^{\circ} ; \varepsilon_{\mathrm{I} 0}=0^{\circ}$ and $5^{\circ}$

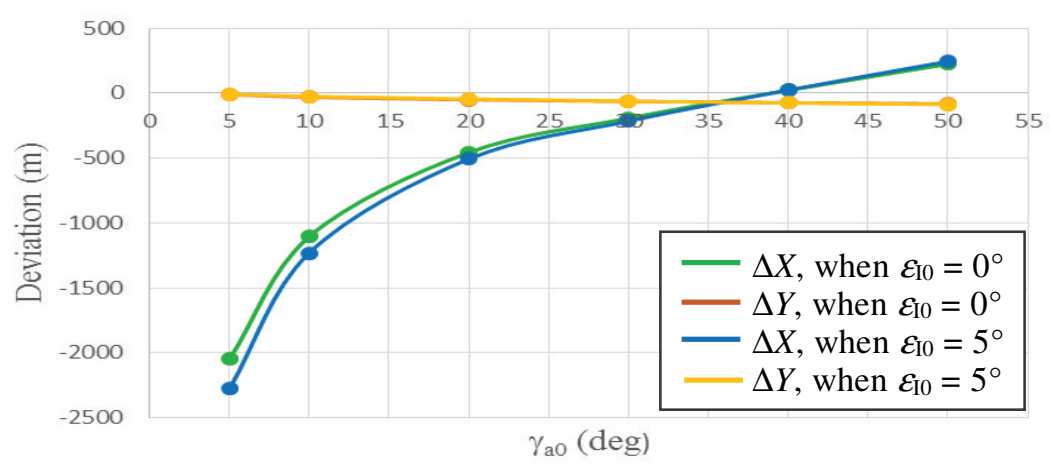

Fig. 19 Dependence of falling point deviation on firing angle when $\lambda_{\mathrm{T} 0}=0.001 \mathrm{rad} ; \varepsilon_{\mathrm{T} 0}=180^{\circ} ; \varepsilon_{10}=0^{\circ}$ and $5^{\circ}$

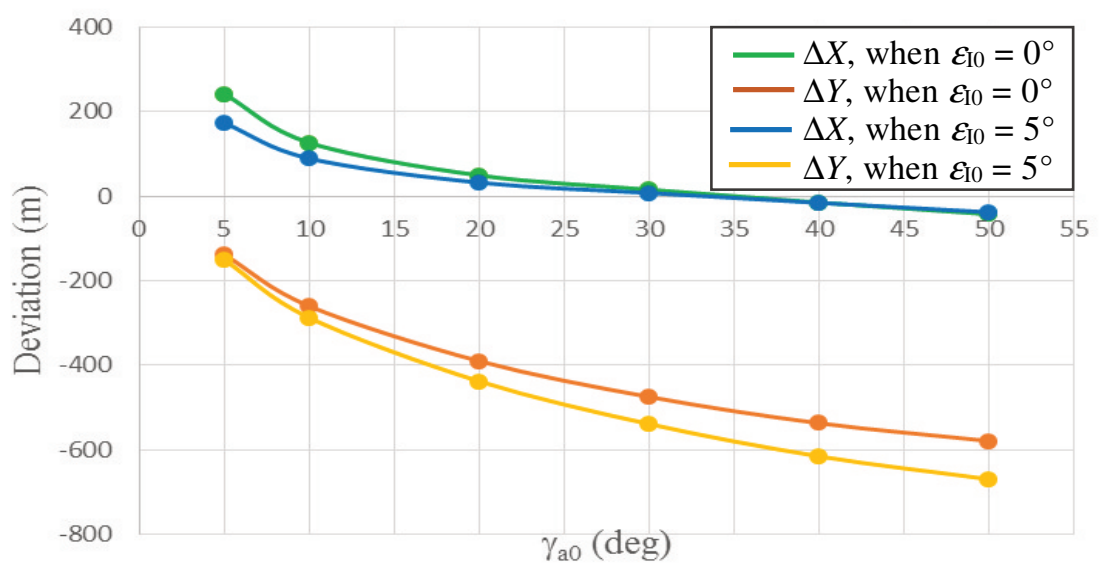

Fig. 20 Dependence of falling point deviation on firing angle when $\lambda_{\mathrm{T} 0}=0.001 \mathrm{rad} ; \varepsilon_{\mathrm{T} 0}=270^{\circ} ; \varepsilon_{10}=0^{\circ}$ and $5^{\circ}$ 
when $\varepsilon_{\mathrm{T} 0}=90^{\circ}$ and $270^{\circ}$, the range deviation decreases. With larger firing angles, the deviations are almost constant.

- At disturbance angles $\mathcal{E}_{\mathrm{T} 0}=0^{\circ}$ and $180^{\circ}$, the difference in lateral deviation is very small, more clearly at the angle of $\varepsilon_{\mathrm{T} 0}=90^{\circ}$ and, on the other hand, it is the greatest at the angle of $\varepsilon_{\mathrm{T} 0}=270^{\circ}$.

\subsection{Combined Effect of CG Offset and Misalignment of Principal Axes of Moments of Inertia}

\section{a) For each firing angle}

This case is the same as the case in 3.5a. The impact of two cases of PAIs misalignment on the effect of CG offset is the same.

b) For variable firing angle

Investigating motions such as those in section 3.2: $\gamma_{\mathrm{a} 0}$ changes; $e_{\mathrm{m} 0}=0.001 \mathrm{~m}$; $\varepsilon_{\mathrm{m} 0}=0^{\circ} ; 90^{\circ} ; 180^{\circ}$ and $270^{\circ}$, but there is an additional disturbance factor as PAIs misalignment angle $\varepsilon_{\mathrm{I} 0}=5^{\circ}$. The results of comparing falling point deviations between motions with and without PAIs misalignment is shown in Figs 21-24.

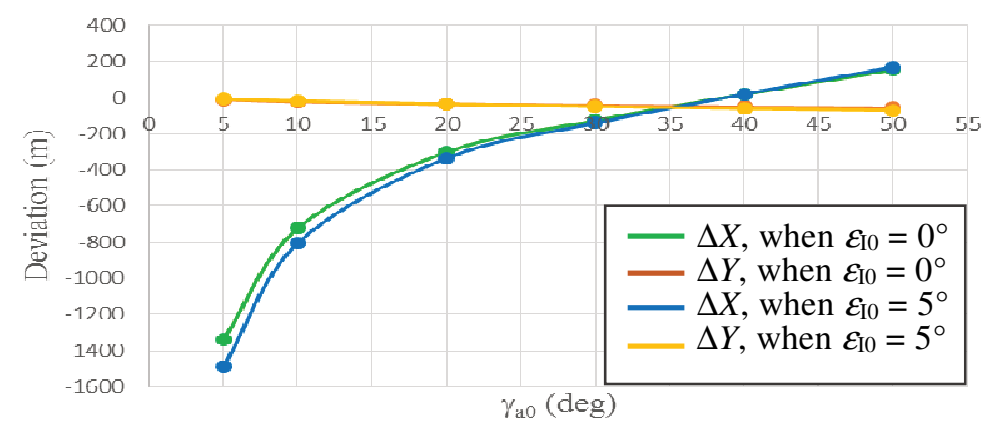

Fig. 21 Dependence of falling point deviation on firing angle when $e_{\mathrm{m} 0}=0.001 \mathrm{~m}$;

$$
\varepsilon_{\mathrm{m} 0}=0^{\circ} ; \varepsilon_{\mathrm{i} 0}=0^{\circ} \text { and } 5^{\circ}
$$

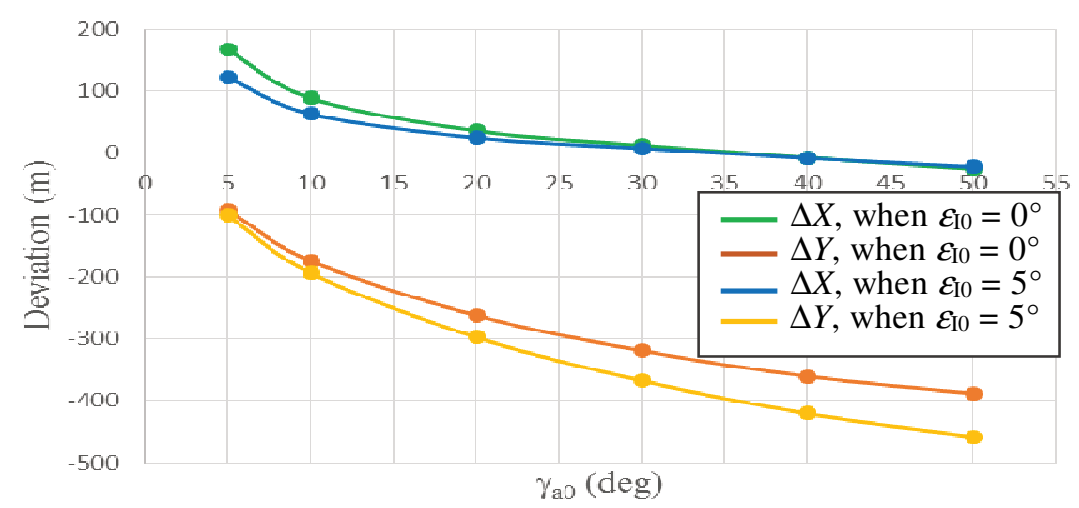

Fig. 22 Dependence of falling point deviation on firing angle when $e_{\mathrm{m} 0}=0.001 \mathrm{~m}$;

$$
\varepsilon_{\mathrm{m} 0}=90^{\circ} ; \varepsilon_{\mathrm{I} 0}=0^{\circ} \text { and } 5^{\circ}
$$




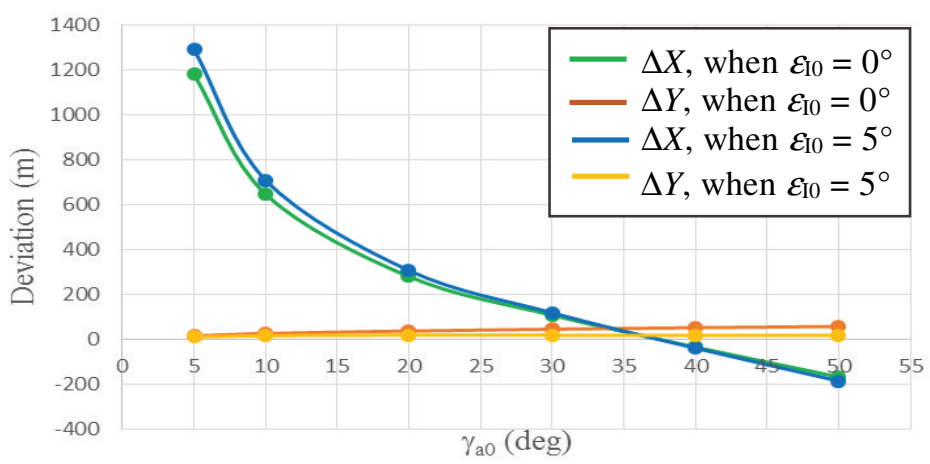

Fig. 23 Dependence of falling point deviation on firing angle when $e_{\mathrm{m} 0}=0.001 \mathrm{~m}$;

$$
\varepsilon_{\mathrm{m} 0}=180^{\circ} ; \varepsilon_{\mathrm{i} 0}=0^{\circ} \text { and } 5^{\circ}
$$

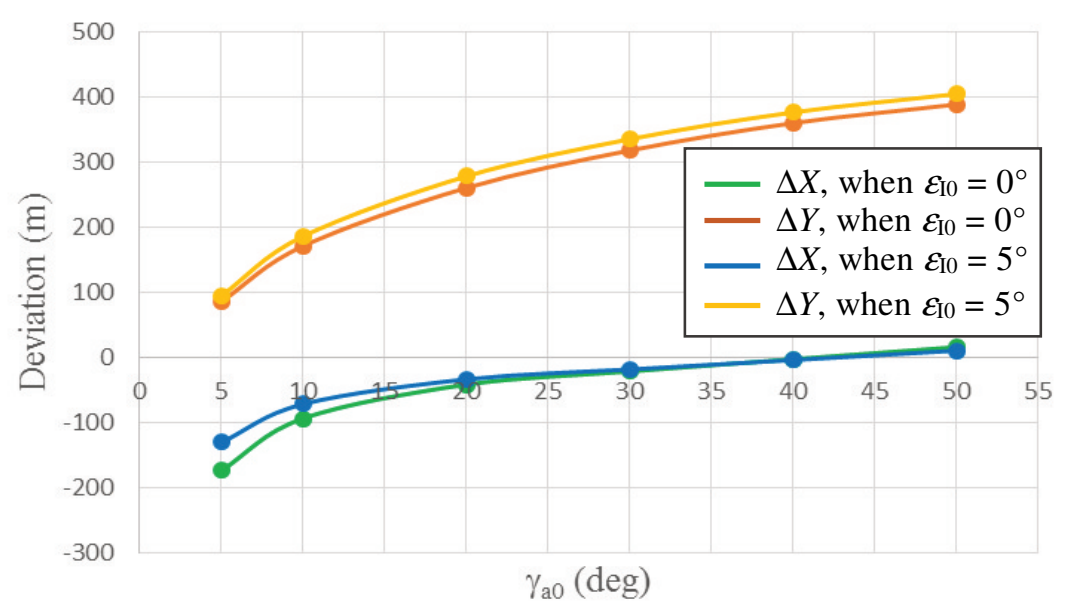

Fig. 24 Dependence of falling point deviation on firing angle when $e_{\mathrm{m} 0}=0.001 \mathrm{~m}$;

$$
\varepsilon_{\mathrm{m} 0}=270^{\circ} ; \varepsilon_{10}=0^{\circ} \text { and } 5^{\circ}
$$

\section{Discussion}

The results in Figs 20 and 23, with explanations similar to section 3.5. show that the impact of PAIs misalignment on the effect of $\mathrm{CG}$ offset in range deviation corresponding to symmetrical disturbance angles $\varepsilon_{\mathrm{m} 0}: 0^{\circ}$ and $180^{\circ} ; 90^{\circ}$ and $270^{\circ}$ are almost the same. With $\varepsilon_{\mathrm{m} 0}=0^{\circ} ; 180^{\circ}$, the range deviation increases, With $\varepsilon_{\mathrm{m} 0}=90^{\circ} ; 270^{\circ}$, the range deviation decreases.

The difference in lateral deviation is very small at the angles of $\varepsilon_{\mathrm{m} 0}=0^{\circ} ; 180^{\circ}$, more clearly at the angle of $\varepsilon_{\mathrm{T} 0}=270^{\circ}$ and it is the greatest at the angle of $\varepsilon_{\mathrm{T} 0}=90^{\circ}$.

\subsection{Combined Effect of Thrust Misalignment, CG Offset and Misalignment of Prin- cipal Axes of Inertia}

In case PAIs misalignment is small, according to sections 3.3 , its effect on the deviations is also quite small. So, the combination of thrust misalignment and mass 
distribution asymmetry is equivalent to the combination between thrust misalignment and CG offset, presented in section 3.4.

With a misalignment angle of $\varepsilon_{10}$ which has a considerable magnitude, the paper investigates the case in which angle $\varepsilon_{10}$ lies on a dangerous plane of the two remaining disturbances (in section 3.4). The impact of PAIs misalignment on the combined effect of the other two factors is assessed through two motions as follows:

The first motion:

- $v_{0}=40 \mathrm{~m} / \mathrm{s} ; \gamma_{\mathrm{a} 0}=30^{\circ} ; \dot{v}_{0}=0.02 \mathrm{rad} / \mathrm{s} ; \alpha_{0}=\beta_{0}=0 ; \dot{\chi}_{\mathrm{a} 0}=\dot{\gamma}_{\mathrm{a} 0}=0$;

- $\lambda_{\mathrm{T} 0}=0.0005 \mathrm{rad} ; \varepsilon_{\mathrm{T} 0}=90^{\circ} ; e_{\mathrm{m} 0}=0.001 \mathrm{~m} ; \varepsilon_{\mathrm{m} 0}=90^{\circ} ; \varepsilon_{\mathrm{l} 0}=0^{\circ}$.

The second motion, $\varepsilon_{10}=5^{\circ}$ in the plane $O X Y$; the remaining parameters are equal to the parameters of the first motion.

The result of comparing the range deviation and lateral deviation of the two motions is shown in Fig. 25.

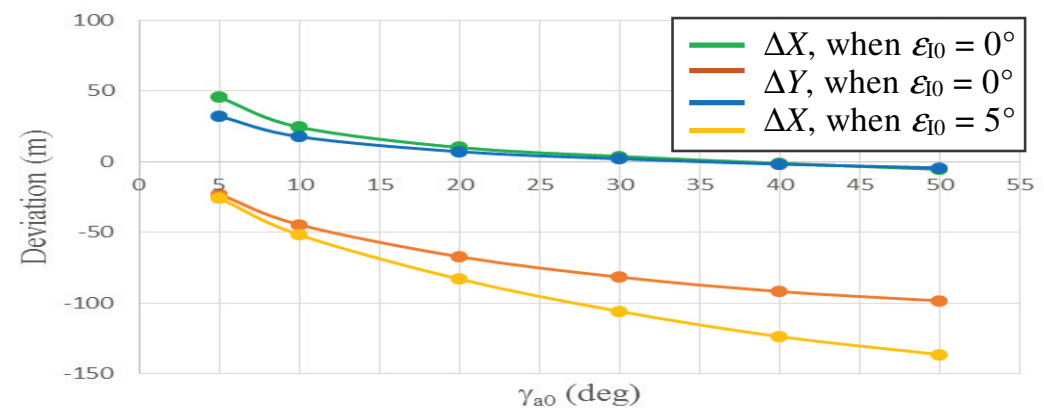

Fig. 25 Dependence of falling point deviation on firing angle when $\lambda_{\mathrm{T} 0}=0.0005 \mathrm{rad}$;

$$
\varepsilon_{\mathrm{T} 0}=90^{\circ} ; e_{\mathrm{m} 0}=0.001 \mathrm{~m} ; \varepsilon_{\mathrm{m} 0}=90^{\circ} ; \varepsilon_{\mathrm{I} 0}=0^{\circ} \text { and } 5^{\circ}
$$

In Fig. 25, the range deviation decreases and the lateral deviation increases. The impact of PAIs misalignment on the combined effect of the remaining two factors is consistent with the results investigated in sections 3.5 and 3.6. The relative position of the two feature points, in this case, corresponds to the cases of $\varepsilon_{\mathrm{T} 0}=270^{\circ}$ in section 3.5 and $\varepsilon_{\mathrm{m} 0}=90^{\circ}$ in section 3.6.

\section{Conclusion}

The paper introduces the development of the mathematical model of rocket motion considering disturbance factors including thrust misalignment, CG offset and PAIs misalignment. The results of solving the model show the laws of the falling point deviation. The most significant outputs of the research can be summarized:

\section{At a firing angle:}

At the positions: $\varepsilon_{\mathrm{T} 0} ; \mathcal{E}_{\mathrm{m} 0}=0^{\circ} ; 90^{\circ} ; 180^{\circ}$ and $270^{\circ}$ when the firing angle increases, the lateral deviation increases too; the range deviation decreases in the interval of the firing angles $\gamma_{\mathrm{a} 0}=5^{\circ} \div 38^{\circ}$, then it increases gradually and changes the sign. For symmetrical disturbance positions through the CG in the values of $\varepsilon_{\mathrm{T} 0}$ and $\varepsilon_{\mathrm{T} 0}+180^{\circ}$, or $\varepsilon_{\mathrm{m} 0}$ and $\varepsilon_{\mathrm{m} 0}+180^{\circ}$, the corresponding deviation graphs are symmetric through 0 -deviation line. 
When considering PAIs misalignment in the plane $O X Y$ or $O X Z$, the change of horizontal-MI has a very small effect on the deviations. The change of longitudinal-MI greatly affects the lateral deviation and a little the range deviation.

For the combined effect of PAIs misalignment with one of the remaining two disturbance factors:

At a firing angle, when the PAIs misalignment changes in the plane $O X Y$ or $O X Z$, its impact on the effect of thrust misalignment (or CG offset) is in two cases the same.

With small firing angles $\left(\leq 25^{\circ}\right)$, PAIs misalignment makes increasing range deviation at the disturbance angles of $\varepsilon_{\mathrm{T} 0}$ or $\varepsilon_{\mathrm{m} 0}=0^{\circ} ; 180^{\circ}$ and reducing range deviation at the disturbance angles $\varepsilon_{\mathrm{T} 0}$ or $\varepsilon_{\mathrm{m} 0}=90^{\circ} ; 270^{\circ}$. With larger firing angles, the deviations are almost constant.

For the combination of thrust misalignment and CG offset:

With the same distance between the two feature points of the two disturbance factors, the deviation graphs get extremums when the two feature points are on the same horizontal or vertical plane.

The combination of three disturbance factors:

It shows that the impact of PAIs misalignment on the combined effect of the other two factors is consistent with its impact on the individual effect of one of the other two.

The results in this paper can serve as a reference for the calculating, designing, and using of unguided rockets.

\section{References}

[1] LE, H.B. and P. KONEČNÝ. Effect of Some Disturbance Factors on the Motion Stability of Unguided Rockets. Advances in Military Technology, 2020, 15(2), pp. 405-423. DOI 10.3849/aimt.01379.

[2] SIOURIS, G.M. Missile Guidance and Control Systems. New York: Springer, 2004. ISBN 978-0-38-700726-7.

[3] KHALIL, M., H. ABDALLA and O. KAMAL. Trajectory Prediction for a Typical Fin Stabilized Artillery Rocket. In: Proceedings of the $13^{\text {th }}$ International Conference on Aerospace Sciences \& Aviation. Cairo: Military Technical College, 2009, ASAT-13-FM-04. DOI 10.21608/ASAT.2009.23742.

[4] NGUYEN, V.T. and D.S. NGUYEN. External Ballistic Textbook (in Vietnamese). Hanoi: Military Technical Academy, 2003.

[5] Fighting Vehicle BM-21 (in Russian) [Technical Specifications]. Moscow: Military Publishing House, 1980. 